\title{
Introduction to the Special Issue on Algorithms and Models for the Web Graph
}

\author{
Anthony Bonato, Ravi Kumar, and D. Sivakumar
}

This issue of Internet Mathematics includes a selection of papers that were presented at the Seventh Workshop on Algorithms and Models for the Web-Graph, WAW 2010, held at Stanford University in December 2010. The papers in this issue, unlike the conference proceedings of the workshop, do not have page limits and contain full versions of proofs and algorithms. All the articles have been thoroughly reviewed in accordance with the usual high standards of Internet Mathematics. The papers address a number of topics related to complex networks such as network-creation games, applications of PageRank, efficient triangle-counting algorithms, and models for online social networks.

The last decade has seen an explosive growth in research on complex networks, ranging from the web graph, to online social networks, to protein-protein interaction networks. Such research has been of great practical importance and has also pushed the frontiers of pure mathematics. One of the goals of the 2010 workshop was to present current research on the theory and applications of complex networks. The papers presented in this special issue should stimulate new and exciting directions in research on complex networks.

We would like to thank the authors and reviewers for making the special issue a reality. 